\title{
NOTES
}

\section{Preparation of Micron-Sized Poly(vinyl acetate) Particles by Suspension Polymerization Using Poly(vinyl alcohol)-Borate Complex Stabilizer}

\author{
Ryoichi Murakami, Hiroshi Hachisako, Kimiho Yamada, \\ and Yoshiaki Motozato \\ Department of Industrial Chemistry, Kumamoto Institute of Technology, \\ Ikeda 4-22-1, Kumamoto 860, Japan
}

(Received July 16, 1992)

\begin{abstract}
KEY WORDS Suspension Polymerization / Poly(vinyl acetate) / Microspheres / Stabilizer / Poly(vinyl alcohol)-Borate Complex /
\end{abstract}

Suspension polymerization generally has been used for the production of polymer particles of diameter in the range of $50-700$ $\mu \mathrm{m}$. Suspension polymerization utilizes an aqueous phase where the immiscible monomer is dispersed by agitation. Polymerization takes place entirely in the monomer phase. The monomer droplets are prevented from coalescing by suspending agents in the suspending phase. As a suspending agent, poly(vinyl alcohol), a very weak surfactant, is often used. ${ }^{1}$ Polymer particles produced in suspension polymerization have applications in chromatographic separations, ion-exchange chromatography, biochemical and bioengineering, etc. In micro high-pressure liquid chromatography swollen porous microparticles of a specific polymer of monodispersity with diameter of $5-10 \mu \mathrm{m}$ are used to exclude certain molecules in a solution by size separation. ${ }^{2}$

The purpose of this study was to prepare microspheres of poly(vinyl alcohol) with diameter of about $10 \mu \mathrm{m}$ as a hydrophilic packing providing a more versatile column packing for aqueous separation. Preparation of the microspheres of poly(vinyl alcohol) from those of poly(vinyl acetate) can be performed by alkaline hydrolysis using methanol in a saturated aqueous solution of sodium sulfate. ${ }^{3}$ Therefore, in this report the conditions for the microspheres of poly(vinyl acetate) were investigated. The reader should recall that the size of the particles produced in suspension polymerization is commonly hundreds of microns in diameter. Here we present a new fact that the production of particles of diameter in the range $1-10 \mu \mathrm{m}$ can be performed by the method of suspension polymerization. This is due to the choice of poly(vinyl alcohol)-borate complex aqueous solution as a suspension stabilizer. Because of the ease of preparation of poly(vinyl acetate) and the control of the degree of hydrolysis, partially hydrolyzed poly(vinyl alcohol) copolymers have been widely used as a suspension stabilizer. It is known that the $88 \mathrm{~mol} \%$ hydrolysis poly(vinyl alcohol) is a more effective stabilizer than the $98 \mathrm{~mol} \%$ grade. ${ }^{4}$ Generally, suspension stabilizers are largely effective when present in interfacial layers between the water and the monomer droplets. Vermeulen et al. ${ }^{5}$ showed that decrease in interfacial tension led to decrease in droplet diameter, and the tendency for interfacial adsorption is more important. The concept of using poly(vinyl alcohol)-borate complex aqueous solution as a suspend- 
ing agent is based on the fact in our previous experiments the surface tension $\left(53 \mathrm{mN} \mathrm{m}^{-1}\right)$ of poly(vinyl alcohol)-borate complex aqueous solution ( $1 \mathrm{wt} \%$ ) showed lower values than that $\left(57 \mathrm{mN} \mathrm{m}^{-1}\right)$ of poly(vinyl alcohol) aqueous solution. ${ }^{6}$

\section{EXPERIMENTAL}

Vinyl acetate was freed from inhibitor by washing with $1 \%$ aqueous solution of sodium hydroxide and distilled. Poly(vinyl alcohol) used as a suspending agent was supplied by Nacalai Tesque Inc. The degree of polymerization (DP) and degree of hydrolysis were about 500 and about $88 \mathrm{~mol} \%$, respectively. Analytical grades of the following chemicals were used directly: sodium tetraborate (borax), calcium carbonate and benzoyl peroxide.

Polymerization was conducted in a $300-\mathrm{ml}$ round-bottomed flask equipped with a agitator and a reflux condenser. Vinyl acetate monomer $(20 \mathrm{~g})$ and benzoyl peroxide $(0.2 \mathrm{~g})$ were added to the flask and stirred to dissolve benzoyl peroxide. Successively poly(vinyl alcohol)-borate complex aqueous solution $(60 \mathrm{ml})$ and calcium carbonate $(1 \mathrm{~g})$ were added to the flask. The temperature was slowly rised to $64^{\circ} \mathrm{C}$ and kept at this for two hours, and then at $80^{\circ} \mathrm{C}$ for 1 hour. The agitation speed was held at $500 \mathrm{rpm}$. The agitation speed was held at $500 \mathrm{rpm}$. The flask was cooled to $30^{\circ} \mathrm{C}$ and the particles obtained were washed by dilute hydrochloric acid and distilled water. The yield was about $60 \%$ the theoretical amount. The size of the particles produced was estimated by light microscopy.

\section{RESULTS AND DISCUSSION}

Figure 1 shows the particle size distributions of poly(vinyl acetate) produced in suspension polymerization using different concentrations of poly(vinyl alcohol) aqueous solution as a suspending agent. High concentrations of stabilizer are associated with particles of lower

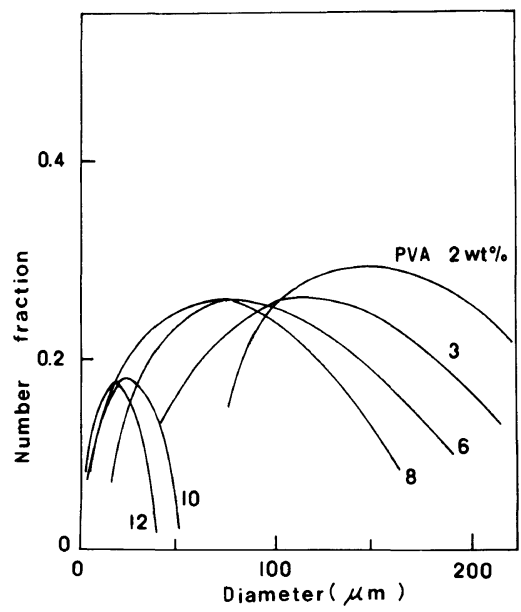

Figure 1. Distributions of diameter of poly(vinyl acetate) microspheres for different concentrations of poly(vinyl alcohol) aqueous solution as a suspension stabilizer.

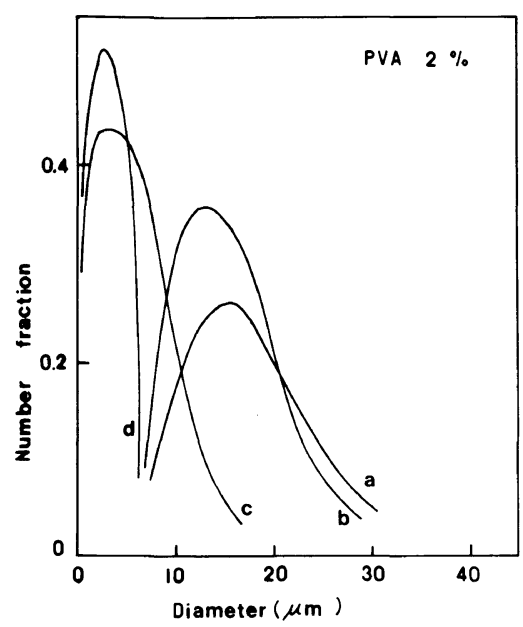

Figure 2. Distributions of diameter of poly(vinyl acetate) microspheres for poly(vinyl alcohol) (2 wt \%)-borate complex aqueous solutions as a suspension stabilizer; concentration of borax: a, $0.02 \mathrm{~mol} \mathrm{l}^{-1} ; \mathrm{b}, 0.04 \mathrm{~mol} \mathrm{l}^{-1} ; \mathrm{c}$, $0.06 \mathrm{~mol} \mathrm{l}^{-1} ; \mathrm{d}, 0.08 \mathrm{~mol} \mathrm{l}^{-1}$.

mean diameter. This may be due to the decrease of interfacial tension with increasing concentration and the increase of the viscosity of a suspending medium. At a concentration of $12 \mathrm{wt} \%$ the products are the particles with diameter in the range $10 \mu \mathrm{m}$ to $50 \mu \mathrm{m}$. Above this concentration, it is difficult to perform suspension polymerization because of high 


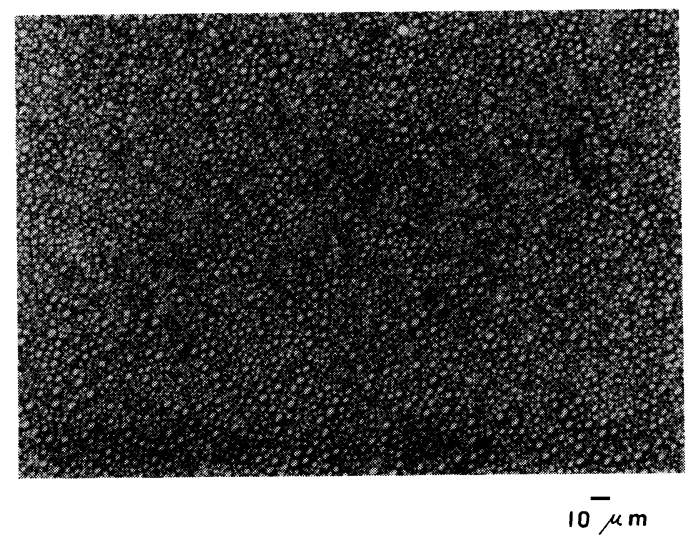

Figure 3. Optical micrograph of poly(vinyl acetate) microspheres produced using a suspension stabilizer consisting of the composition of poly(vinyl alcohol) concentration $2 \mathrm{wt} \%$ and borax concentration 0.08 mol $1^{-1}$.

viscosity, and so this method can not produce particle size in the range $1-10 \mu \mathrm{m}$. In the case of using poly(vinyl alcohol)-borate complex aqueous solution as a suspending agent, particle size obtained decreased strikingly. Figure 2 shows the distribution curves of particles for the poly(vinyl alcohol)-borate complex aqueous solutions (poly(vinyl alcohol) concentration: $2 \mathrm{wt} \%$, the amount of borax added: $0.02,0.04,0.06,0.08 \mathrm{~mol} \mathrm{l}^{-1}$ ) as a stabilizer. In this experiment reproducible distribution curves were obtained. It is clear that under comparable conditions high concentrations of borax are associated with particles of lower mean diameter. At a concentration of borax $0.08 \mathrm{moll}^{-1}$ the particles with diameter $1-10 \mu \mathrm{m}$ were produced. Beyond this concentration, the experiment resulted in failure because of the adhesion of partially polymerized particles during polymerization. Moreover, in the case of poly(vinyl alcohol) with above DP $=1000$ and $98 \mathrm{~mol} \%$ hydrolysis the experiment was also unsuccesful because of gelation of the solution. In the case of increasing the concentration of poly(vinyl alcohol) to $3 \mathrm{wt} \%$, particles with diameter in the range $1-15 \mu \mathrm{m}$ were produced in the range of borax concentration $0.02-0.04$ mol $1^{-1}$. The shape of the distribution curve for particle size is sharper and closer to monodispersity when using a suspension stabilizer consisting of poly(vinyl alcohol) concentration $2 \mathrm{wt} \%$ and borax concentration $0.08 \mathrm{~mol}^{-1}$. It is thus clear that the production of particle size in the range $1-10 \mu \mathrm{m}$ is possible with a suitable stabilizer, for example, poly(vinyl alcohol)-borate complex aqueous solution with the concentration of poly(vinyl alcohol $)(\mathrm{DP}=500,88 \mathrm{~mol} \%$ hydrolysis $) 2 \mathrm{wt} \%$ and borax $0.08 \mathrm{moll}^{-1}$. A micrograph of the particles of poly(vinyl acetate) prepared under the above conditions is shown in Figure 3. The use of poly(vinyl alcohol) borate complex aqueous solution as a suspending agent in suspension polymerization was very effective for the preparation of polymer particles in the size range between suspension and emulsion polymers, i.e., about $10 \mu \mathrm{m}$.

Acknowledgment. The authors thank Dr. H. Ishibashi (Chisso Co., Ltd.) for many useful discussions.

\section{REFERENCES}

1. J. V. Dawkins in "Comprehensive Polymer Science," Vol. 4, "Aqueous Suspension Polymerizations," S. S. Allen and J. G. Bevington, Ed., Pergamon Press, Oxford, U.K., 1989, p 231.

2. K. H. Altgelt in "Advances in Chromatography," Vol. 7, Dekker, New York, N.Y., 1968, Chapter 3.

3. Y. Motozato and C. Hirayama, Nippon Kagaku Kaishi, 1087 (1972).

4. J. M. G. Lankveld and J. Lyklema, J. Colloid Interface Sci., 41, 454, 460, 475 (1972).

5. T. Vermeulen, G. M. Williams, and G. E. Langlois, Chem. Eng. Prog., 51, 85 (1955).

6. R. Murakami, Rep. Progr. Polym. Phys. Jpn., 26, 71 (1983). 